\title{
Gerichtliche Vorgaben für die ärztliche Erstabklärung
}

\author{
Das Bundesgericht hält fest, dass die Beurteilung des natürlichen Kausalzu- \\ sammenhangs zwischen dem Unfallereignis und den geklagten organisch \\ nicht hinreichend nachweisbaren Beschwerden aufgrund einer eingehenden \\ medizinischen Abklärung zu erfolgen habe. Im Grundsatzentscheid BGE 134 \\ V 109 vom 19. Februar 2008 werden die konkreten Anforderungen an eine \\ genügende ärztliche Erstabklärung bei Verdacht auf ein kraniozervikales \\ Beschleunigungstrauma beschrieben.
}

David Weiss *

\footnotetext{
* Der Autor ist Direktionsmitglied der «Zürich» Versicherungs-Gesellschaft und Richter am Strafgericht des Kantons Basel-Landschaft. Die Meinung des Autors deckt sich mit derjenigen des medizinischen Dienstes des Schweizerischen Versicherungsverbandes.
}

Korrespondenz:

lic. iur. David Weiss

Zürich Versicherungs-Gesellschaft

Komplexe Körperschäden Mitte

Alpenstrasse 6

Postfach

CH-6002 Luzern
Das Bundesgericht hält in seinem Grundsatzentscheid BGE 134 V 109 fest, dass der Unfallhergang und die anschliessend auftretenden Beschwerden möglichst genau und verifizierbar zu dokumentieren sind. Der erstbehandelnde Arzt ist gehalten, die versicherte Person sorgfältig abzuklären (in der Regel eingehende Befragung sowie klinische und gegebenenfalls röntgenologische Untersuchungen). Zur sorgfältigen Erstabklärung gehört auch die Befragung der versicherten Person nach ihrem gesundheitlichen Vorzustand, so unter anderem nach psychischen Beschwerden vor dem Unfall oder im Zeitpunkt des Unfalls. Die Aussagen der versicherten Person zum Unfallhergang und zu den bestehenden Beschwerden sind gestützt auf die erhobenen Befunde und weitere zur Verfügung stehende Angaben und zum anschliessenden Verlauf kritisch zu prüfen. Gelangt der Arzt bei der Diagnosestellung zur Auffassung, eine der zur Diskussion stehenden Verletzungen komme aufgrund der bis dahin gegebenen zuverlässigen Anhaltspunkte nur als Verdachts- oder Differentialdiagnose in Frage, hat er dies in seinem Bericht so zum Ausdruck zu bringen. Das Bundesgericht hält weiter fest, es sei von besonderer Bedeutung, dass der Arzt in seinem Bericht, gegebenenfalls in dem vom Schweizerischen Versicherungsverband (SVV) empfohlenen «Dokumentationsbogen für Erstkonsultation nach kraniozervikalem Beschleunigungstrauma» (früher gebräuchlich: Zusatzfragebogen für HWSVerletzungen), nebst den weiteren der Diagnosestellung zugrunde gelegten Überlegungen auch den Verlauf der Beschwerden ab dem Unfallzeitpunkt genau beschreibt. Zudem hat er gegebenenfalls bestehende Anhaltspunkte für einen verzögerten Verlauf und/oder ein Chronifizierungsrisiko anzuzeigen.

\section{Directives du Tribunal fédéral pour le premier examen médical}

Le Tribunal fédéral stipule que le lien naturel de causalité entre l'accident et les douleurs organiques ressenties et ne pouvant pas être suffisamment prouvées doit être évalué sur la base d'un examen médical approfondi. Les exigences concrètes posées à un premier examen médical suffisant en cas de soupçon d'un traumatisme cranio-cervical par accélération sont décrites dans l'arrêt ATF 134 V 109 du 19 février 2008.

Der richterliche Hinweis auf die kritische Prüfung ist schon deshalb sinnvoll, weil es wiederholt zu Verurteilungen wegen Schleudertraumabetrug gekommen ist (vgl. Urteil des Bundesgerichts 6B 188/2007 vom 15. August 2007; Urteil des Bundesgerichts 6B 299/2007 vom 11. Oktober 2007).

\section{Erwägungen zur Arbeitsunfähigkeit}

Zur Frage der Arbeitsunfähigkeit hat das Bundesgericht sinngemäss ausgeführt, dass bei leichten bis mittelschweren Schleudertraumen der HWS ein längerer oder gar dauernder Ausstieg aus dem Arbeitsprozess als eher ungewöhnlich erscheine. Bereits das Eidgenössische Versicherungsgericht hat in einem konkreten Einzelfall unter Berücksichtigung der unfallbedingten Einschränkungen (belastungs- und bewegungsabhängige Kopf- und Nackenschmerzen, eingeschränkte Beweglichkeit von Kopf und Halswirbelsäule, rasche Ermüdbar- 
keit) jede leichte bis mittelschwere Tätigkeit ohne Notwendigkeit von Überkopfarbeiten, vorgeneigtem Stehen und des Hebens oder Tragens schwerer Lasten als grundsätzlich zumutbar bezeichnet (vgl. Urteil des EVG U 54/05 vom 19. Juli 2005, Erw. 2.1).

In Konkretisierung der Schadenminderungsobliegenheit verlangt nun das Bundesgericht im Grundsatzentscheid vom Versicherten ernsthafte Arbeitsversuche trotz allfälliger persönlicher Unannehmlichkeiten.

\section{Weiterer Grundsatzentscheid zu den diagnostischen Mitteln}

Das Bundesgericht hat zwischenzeitlich in einem weiteren Grundsatzentscheid sinngemäss ausgeführt, die funktionelle Magnetresonanztomographie (fMRT) stelle nach dem heutigen Stand der medizinischen Wissenschaft kein geeignetes Beweismittel zur Beurteilung der Unfallkausalität von Beschwerden nach HWS-Traumen dar (vgl. Urteil des Bundesgerichts 8C 152/2007 vom 26. Mai 2008, Erw. 5.4).

\section{Die Bedeutung von BGE 134 V 109}

Die vom Bundesgericht als Entscheidungsgrundlage verlangte sorgfältige ärztliche Erstabklärung mit eingehender Befragung - unter Einbezug von Fragen nach dem Vorzustand - verlangt von der Ärztin in einem sehr kurzen Zeitraum die Ermittlung und Dokumentierung einer Vielzahl von detaillierten Informationen, die von grosser Wichtigkeit sind.

Der Arzt trägt mit der korrekten Erhebung und Dokumentation der «Aussagen der ersten Stunde» eine grosse Verantwortung. Das Bundesgericht hat sinngemäss ausgeführt, bei sich wider- sprechenden Angaben des Versicherten über den Unfallhergang sei auf die Beweismaxime hinzuweisen, wonach die sogenannten spontanen «Aussagen der ersten Stunde» in der Regel unbefangener und zuverlässiger seien als spätere Darstellungen, die bewusst oder unbewusst von nachträglichen Überlegungen versicherungsrechtlicher oder anderer Art beeinflusst sein könnten. Wenn der Versicherte seine Darstellung im Laufe der Zeit wechsle, komme den Angaben, die er kurz nach dem Unfall gemacht habe, meistens grösseres Gewicht zu als jenen nach Kenntnis einer Ablehnungsverfügung des Versicherers (vgl. BGE 121 V 45, 47, Erw. 2a) mit Hinweisen).

Um die Erstabklärung systematisch durchzuführen, empfiehlt es sich mit Blick auf den Grundsatzentscheid noch mehr, mit dem Dokumentationsbogen für Erstkonsultation nach kraniozervikalem Beschleunigungstrauma zu arbeiten. Dieser Bogen kann als Word- oder PDFFormular auf www.med.svv.ch heruntergeladen werden. Mit diesem checklistenartigen Erfassungsinstrument, das sowohl als Fragebogen für den Unfall- und Krankenversicherer als auch für die eigene Dokumentation in der Krankengeschichte dient, steht bereits heute eine gute Orientierungshilfe zur Verfügung. Der medizinische Dienst des SVV prüft gegenwärtig die Anpassung des bestehenden Dokumentationsbogens aufgrund der neuen höchstrichterlichen Vorgaben.

Nicht durchgeführte ernsthafte Arbeitsversuche wirken sich für den Versicherten nachteilig auf die rechtliche Adäquanzbeurteilung aus. Auch unter diesem rechtlichen Aspekt ist mit dem Patienten auf den ersten medizinisch vertretbaren Zeitpunkt ein Arbeitsversuch zu vereinbaren. 